\title{
Zur Geschichte der Magenresektion
}

\author{
Von Juraj Körbler und Alexander Haeffner
}

In der Geschichte der Magenresektion kommt der von Finsteren angegebenen Modifikation des von BıLlnotн eingeführten Verfahrens eine große Bedeutung zu. Die Methode hat sich in den fünfzig Jahren, seitdem sie angegeben worden ist, vielfach bewährt. Sie bildet gewissermaßen den Abschluß einer langen Entwicklung.

Die Technik der Magenresektion ist vornehmlich zur Behandlung des Magenkrebses ausgearbeitet worden, und die meisten dabei beteiligten Chirurgen hatten dieses Ziel vor Augen.

Bereits im Jahre 1810 hat Daniel Carl Theodor Merrem (1790-1859) experimentell nachgewiesen, daß man den Pylorus resezieren kann (Animadeversiones quaedam chirurgicae experimentis in animalibus factis illustratae, I.D. Gießen 1810). Die Anregung gab ein amerikanischer Arzt aus Philadelphia, dessen Freund Dr. Midleton an Pyloruskarzinom gestorben war. Merrem hat bei einem Hund, der zehn Stunden gehungert hatte, am 19. November 1809 den Pylorus reseziert. Der Hund verendete am 8. Dezember. Ein zweiter Hund, den er am 8. Dezember operiert hatte, lebte bis zum 24. Janaur 1810. Ein dritter Hund wurde am 5. März 1810 operiert, verendete jedoch bereits am folgenden Tage.

Am 9. April 1879 hat in Paris Jules Emile PéAN (1830-1898) eine Magenresektion beim Menschen ausgeführt, veranlaßt durch die Mittelung eines Arztes aus Arras, der einige Jahre vorher eine solche Operation unternommen hat. Der von PÉAN operierte Kranke überlebte die Operation nur um zwei Tage. RyDYGIER hat in Deutschland am 14. November 1880 eine Pylorusresektion beim Menschen versucht (Arch. klin. Chir. 26 [1881] 731). Der Kranke starb einige Stunden nach der vierstündigen Operation. Am 29. Januar 1881 ist Christian Albert Theodor Billroth (1829-1894) in Wien bei einer 43jährigen Frau die Resektion des Pylorus gelungen (Wien. med. Wsch. 1881, 162). Der Pylorus war durch ein Karzinom vollständig stenosiert. Die Operation dauerte 90 Minuten. Die Kranke starb vier Monate nach der Operation an Lebermetastasen. Die Technik dieser Operation wird als Billroth I bezeichnet: Der Magenrest wird mit dem Duodenum vereinigt.

Als Palliativverfahren beim inoperablen Pyloruskarzinom hat ANTon WöLFLER (1850-1917), Assistent von Billroth, am 28. September 1881 die von ihm als Gastroenterostomie bezeichnete Operation eingeführt (Z. Chir. 1881, Nr. 15). Dabei wird nur der Magen eröffnet und mit einer Darmschlinge verbunden. Die als Billroth II bezeichnete Operation hat BILLRoth am 15. Januar 1885 ausgeführt. Bei der an Magenkrebs leidenden Kranken wurde zuerst eine Gastroenterostomie nach WöLfler angelegt. Danach ist der erkrankte pylorische 
Magenteil entfernt worden, und die Öffnungen am Duodenum sowie am Magen wurden vernäht und verschlossen. Die Kranke lebte vier Monate nach der Operation. Bei der Autopsie fand man Metastasen und ein Rezidiv an der Anastomose.

Die von ihm angegebene Modifikation der als Billroth II bezeichneten Magenresektion hat HANS Finsterer (1877-1956) am 20.September 1911 ausgeführt (Dtsch.Z.Chir.128 [1914] 514). Wegen eines ausgebreiteten Magenkrebses, der fast bis zur Kardia reichte, hat er vier Fünftel des Magens reseziert. Nach der Resektion hat er den oberen Teil des Magenlumens verschlossen und den unteren Teil hinter dem Colon mit der ersten Jejunum-Schlinge durch eine End-zuSeit-Anastomose verbunden.

In den Jahren 1945-1948 hat Finsteren an seinem Buch über die Chirurgie gearbeitet, und einer von uns (HAEFfner) hat damals der Aufforderung, sich an dieser Arbeit zu beteiligen, bereitwillig entsprochen. Es wurden jedoch nur etwa 350 Seiten ausgearbeitet, von denen ein Teil im Jahre 1952 als Chirurgie des Dickdarms im Verlag Maudrich in Wien erschienen ist. Der Rest, in welchem auch die Beschreibung der nach Finsterer benannten Magenresektion enthalten ist, verblieb als maschinengeschriebenes Manuskript.

Auf Seite 70 und 71 dieses Manuskriptes hat Finsteren seine Methode der Magenresektion beschrieben und auch ihre Benennung begründet:

"Als ich im September 1911 an der Klinik Hochenegg bei einer ganz herabgekommenen Frau (Gewicht 371/2 kg) wegen eines infiltrierten Carcinoms eine subtotale Resektion ausführte, war es absolut unmöglich, die Methode Billroth II, so wie wir sie gelernt hatten, anzuwenden, also den Magen ganz zu verschließen und eine Seit-zu-Seit-Anastomose mit dem schlauchförmigen Magenrest anzulegen. Daher habe ich aus Not das einzig Mögliche gemacht: Ich habe den oberen Teil der großen Magenöffnung verschlossen und den unteren Teil End zu Seit in die erste Jejunum-Schlinge eingepflanzt. Der Bedeutung halber soll die Krankengeschichte dieses Falles, der die Wiege dieser wichtigen Modifikation geworden ist, mitgeteilt werden:

60 jährige Frau, seit fünf Monaten Magenbeschwerden, Drücken nach dem Essen, Appetitlosigkeit, hochgradige Abmagerung. Seit vier Wochen bemerkt die Patientin selbst eine Geschwulst im Bauch, sie wird daher an die Klinik Hochenegg geschickt. Befund: sehr magere kachektische Frau, Gewicht $37 \frac{1}{2} \mathrm{~kg}$ bei normaler Größe. In der Oberbauchgegend faustgroßer, beweglicher Tumor palpabel. Probefrühstück ergibt keine freie Salzsäure, keine Milchsäure. Operation am 19. September 1911 in Lokalan. der Bauchdecken und Mesent. An. mit 1/4 prozentiger Novocainlösung und $30 \mathrm{~cm}^{3}$ Äther zum Absuchen des Bauches. Infiltrierendes Carcinom der kleinen Kurvatur und Vorderwand des Magens, bis in den Oesophagus reichend, mit der Leber entzündlich verklebt, Mesocolon transversum durchwuchert; vergrößerte Drüsen bis zum Oesophagus reichend, einige derselben vereitert. Resektion des Magens, bis zum Oesophagus sich erstreckend, Resektion des Mesocolon. Es bleibt nur ein ganz schmaler Magenrest, an dem eine typische Anastomose nach Billroth II unmöglich ist. Daher wird aus Verlegenheit der obere Teil der Magenwunde 
verschlossen, durch die nach der Resektion des Mesocolons entstandene Öffnung die erste Jejunum-Schlinge in die Oberbauchgegend gezogen, das Mesocolon an die hintere Magenwand genäht und dann der offen gebliebene Teil der Resektionsfläche des Magens mit der ersten Jejunum-Schlinge End zu Seit anastomosiert, schließlich der rechte Rand des Mesocolon-Schlitzes auf die vordere Magenwand fixiert. Tamponade der Abszeßhöhle (vereiterte Drüsen). Bauchnaht. Operationsdauer 2 $\frac{1}{2}$ Stunden. Histologischer Befund (pathologisches Institut): Tubuläres Carcinom.

Idealer postoperativer Verlauf. Anfangs Diarrhöen, die aber auf Salzsäure und Opium stehen. Nach Entfernung der Tamponade Radiumbestrahlung durch die Fistelöffnung. Am 26. Oktober 1911 wird die Patientin nach $5 \mathrm{~kg}$ Gewichtszunahme geheilt entlassen, sie kann nur wenig essen (Beschwerden des kleinen Magens).

Wegen des direkt idealen postoperativen Verlaufs habe ich von da ab in allen Fällen diese End-zu-Seit-Anastomose ausgeführt. Da ich durch Umfrage bei den Kollegen erfuhr, daß diese noch immer die Originalmethode Billroth II anwenden, so habe ich auf der Naturforscherversammlung in Wien 1913 über die Methode kurz berichtet. In der Aussprache teilte mir v. Haberen mit, daß Hofmeister die gleiche Modifikation des Billroth II mache, ohne den Ort der Publikation näher anzugeben. Erst auf eine Anfrage bei Hofmeister erfuhr ich, daß BuRK 1911 in seiner Arbeit über die chirurgische Behandlung gutartiger Magenerkrankungen die Methode kurz beschrieben hat, ohne daß sie dadurch überhaupt bekannt wurde. In meiner ausführlichen Arbeit über die Technik der Magenresektion habe ich daher die Methode ,Modifikation nach HofmeisterFinsterer ${ }^{6}$ genannt. Seit dieser Zeit habe ich selbst die Methode in mehr als... Fällen immer in der gleichen Weise ausgeführt, sie wurde auch von meinen Schülern in über ... Fällen ebenso gemacht.»

So weit Finsterer über seine Methode. Die statistischen Angaben fehlen leider. In seiner Arbeit «Zur Klinik und chirurgischen Behandlung des Magenkrebses» (Arch.klin.Chir.159 [1930] 30) erwähnt Finsterer, daß diese seine Operation in Wien als «Methode Krönlein-Mikulicz» bezeichnet wird. Er bemerkt dazu, daß es durchaus nicht notwendig ist, die Modifikation mit einem eigenen Namen zu belegen, man kann sie ebensogut nach dem Vorschlag von NARATH als «Billroth II cum gastroenterostomia retrocolica oralis inferior » bezeichnen, ein allerdings langer Ausdruck. Seiner Ansicht nach ist es aber ganz unrichtig, die Methode als «Krönlein-Mikulicz» zu bezeichnen, denn wenn man diese Namen schon beibehält, dann muß man die Methode mit allen ihren Nachteilen ebenso ausführen, wie sie Mikulicz im Handbuch der Chirurgie selbst beschrieben hat. Dazu ist jedoch zu betonen, daß heutzutage für die von Finsterer beschriebene Operation die Bezeichnung «Hofmeister-Finsterer» allgemein angenommen worden ist. Unter dieser Bezeichnung ist die vor einem halben Jahrhundert von Finsterer angegebene Operation Gemeingut der Chirurgen geworden, und die eigene Darstellung dieser Operation vom Altmeister der Chirurgie soll nicht der Vergessenheit anheimfallen. 